CBP Note 545

LBNL Note 54096

pbar Note 684

\title{
Aperture Studies for the AP2 Anti-Proton Line at Fermilab
}

\author{
Ina Reichel, Michael Zisman and Massimo Placidi \\ Lawrence Berkeley National Laboratory \\ Berkeley, CA 94720
}

December 5, 2003

\begin{abstract}
The AP2 beamline transports anti-protons from the production target to the Debuncher ring. For many years the observed aperture has been smaller than that estimated from linear, on-energy optics. We have investigated possible reasons for the aperture restriction and have identified several possible sources, including residual vertical dispersion from alignment errors and chromatic effects due to very large chromatic lattice functions. We discuss the possible sources, suggest some remedies, and propose specific studies, where needed, to evaluate suspected problems.
\end{abstract}

\section{Introduction}

The AP2 beam line at Fermilab transports anti-protons from the target where they are produced to the Debuncher ring. To first order the distribution of the particles is flat transversely and longitudinally. In addition the beam consists only to about $1 \%$ of anti-protons. The remainder are pions and other particles which decay along the line and during the first turns in the Debuncher. This complicates measurements of beam parameters. The 
first element of the line is a lithium lens to focus the beam. The lithium lens is not part of the studies presented here.

The line was originally designed to have enough aperture for a nominal emittance of $20 \pi \mathrm{mm}$ mrad in both transverse planes and an energy acceptance of $\pm 2.0 \%$ [1]. The physical aperture of the Debuncher components, with some exceptions, is known to be at least $40 \pi \mathrm{mm}$ mrad for a beam with a $\pm 2.0 \%$ energy spread [2]. The measured admittance of the Debuncher is $24.7 \pi \mathrm{mm}$ mrad (horizontal) and $19.6 \pi \mathrm{mm} \mathrm{mrad}$ (vertical). The reason for the discrepancy is believed to be the limited control of the closed orbit in the Debuncher [2] which will be improved in the near future by putting quadrupole magnets on movers and thus using them as orbit correctors.

Measurements show a transmission of only $20 \pi \mathrm{mm}$ mrad in the horizontal and $12 \pi \mathrm{mm}$ mrad in the vertical plane [3]. As the admittance of the Debuncher is larger than that, attempts have been made to increase the admittance of AP2, therefore increasing the number of transmitted antiprotons [4].

The problem has existed for a while and an earlier study identified the aperture in the septum as a possible bottleneck [5]. However replacing the septum by one with a larger aperture and some other modifications suggested in [5] did not improve the situation [2]. In this study, other possible sources of transmission restrictions have been investigated.

If the aperture of the line could be increased to accept an emittance of $40 \pi \mathrm{mm}$ mrad in both planes this would double the anti-proton yield into the Debuncher, which, in turn, would lead to a significant gain in the overall performance of the Tevatron.

\section{Lattice Studies}

So far, studies investigating the restricted aperture only considered linear, on-energy optics and no imperfections. We have looked at machine imperfections like alignment and field errors and also off-momentum optics, which are important as the energy spread of the beam is large. 


\subsection{Nominal Lattice}

Figure 1 on the following page shows the lattice functions for the nominal lattice ${ }^{1}$. From this one can calculate the beam envelopes and compare them to the apertures. This is shown in Fig. 2 on the next page. One can see that in the area around the momentum collimator (which is fully open), located near $170 \mathrm{~m}$ at the peak of the horizontal dispersion, the beam is scraping slightly in the horizontal plane. The apertures used for the bending magnets (the aperture restriction near $30 \mathrm{~m}$ and the longer aperture restrictions between 150 and $190 \mathrm{~m}$ ) are upper limits. We do not yet have final numbers for the apertures of the vacuum chamber in those areas. The numbers used are rough estimates (error of $\pm 2 \mathrm{~cm}$ ) taken from scanned versions of the technical drawings. However they include the fact that the aperture is smaller than the physical aperture of the chamber because the chamber is straight in the magnets although some of them bend the beam by several degrees and therefore the sagitta is not negligible.

The lattice used for the studies presented here is the one that has been optimized for an emittance of $25 \pi \mathrm{mm}$ mrad. The main difference between this lattice and a lattice optimized for $40 \pi \mathrm{mm}$ mrad is the matching with the lithium lens, which results in different initial Twiss parameters for the line. This is compensated by rematching the line using the first six quadrupole magnets. The main reason for using this lattice in our studies is that this is the lattice that is currently used in the machine, and we wish initially to understand why it leads to the observed aperture restrictions. Aside from some rematching at the upstream end, the optimized $40 \pi \mathrm{mm}$ mrad lattice is not very different from that depicted in Fig. 1 .

The lattice functions, and therefore the envelopes, in the real machine will be different from the nominal ones. This is caused by machine errors such as alignment or field errors and by a mismatch of the incoming beam. The different sources of errors have been studied to determine their influence on the aperture and are discussed below. Also particles with an energy difference to the nominal energy will see a different lattice. The chromatic functions also contribute to a diminished aperture as the energy spread of the beam is significant and flat over the acceptance of the line.

\footnotetext{
${ }^{1}$ It was discovered very recently, that in the translation of the lattice file from OptiM to MAD the sign of the vertical bending magnets was inverted. Therefore the vertical dispersion has the wrong sign. We do not believe that this has a large impact on the results shown in this paper.
} 


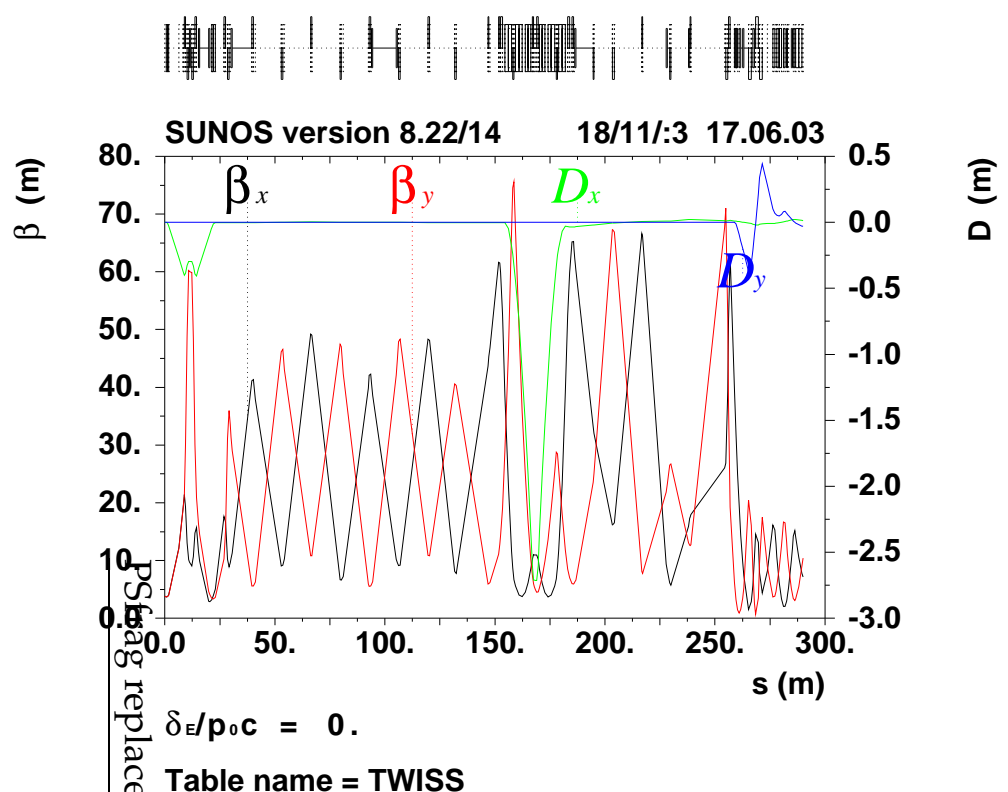

Figure 1: Nominal lattice of the AP2 beam line calculated with MAD [6].
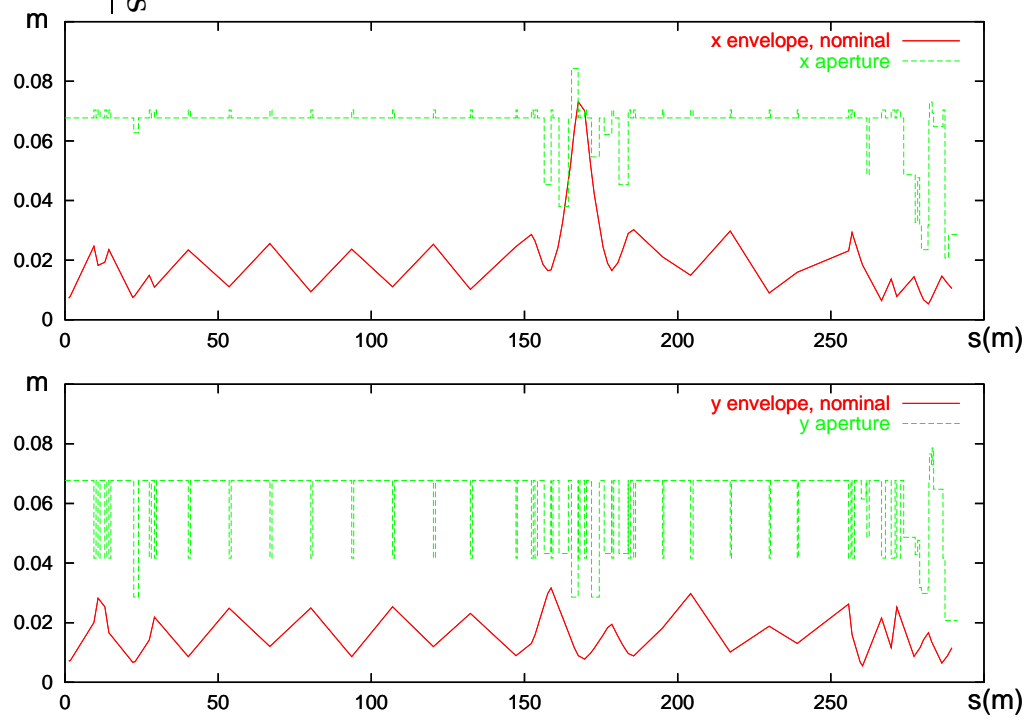

Figure 2: Beam envelopes (in $\mathrm{m}$ ) for $40 \pi \mathrm{mm}$ mrad emittances and $4.5 \%$ full width energy spread based on the $\beta$-functions and dispersions shown in Fig. 1. Aperture restrictions, where known, are indicated. Betatron and dispersion contributions are added linearly as all distributions are (to first order) flat. 


\subsection{Mismatch of the Lithium Lens}

In case of a mismatch of the lithium lens, the initial lattice functions are wrong, which leads to a mismatch down the line. Also, the upstream beam line (the lithium lens and/or the proton beam producing the anti-protons) can have a transverse offset with respect to the AP2 line axis, leading to an offset of the beam. We discuss each of these separately:

\subsubsection{Strength Error}

A strength error of the lithium lens was studied. For a strength error of $5 \%$ one gets a $\beta$-beat of $50 \%$ but the envelopes are still inside the aperture. The longitudinal position of the lens is optimized on anti-proton yield. Therefore we can assume that the error due to a mismatch is much smaller and therefore should not be a problem.

\subsubsection{Transverse Offset}

If the particles start down the line with a transverse offset or angle of more than a few tens of $\mathrm{mm}$ or a few mrad, the orbit excursions become very large (order of $10-20 \mathrm{~cm}$ ). In practice, due to the size of the lithium lens, any possible errors are much smaller than the ones assumed here. We therefore assume that any possible transverse offset does not cause aperture problems.

\subsection{Mismatch into the Debuncher}

If either the $\beta$-functions or the dispersion are not matched properly going into the Debuncher this could lead to losses in the Debuncher. We therefore compared lattice functions at the end of the line to the lattice functions in the Debuncher ring at the appropriate place. Table 1 shows the lattice parameters calculated for each lattice ${ }^{2}$.

The difference in dispersion is of the order of residual dispersion due to machine errors so this is probably no cause for worry. However there is a significant mismatch in $\beta$-functions which leads to a $50 \% \beta$-beat in the Debuncher if one projects the $\beta$-functions from AP2 into the Debuncher. This

\footnotetext{
${ }^{2}$ There are still inconsistencies between the two lattice files. One of the common magnets is at a different position in each of them and also the strengths of some of the common quadrupole magnets differ. Also one lattice uses effective lengths for the magnets whereas the other uses the length of the yoke. Work is going on to reconcile the lattice files with each other, with available technical drawings and with measurements in the tunnel [7].
} 
Table 1: Lattice functions at the end of the injection kicker calculated using the lattice information for AP2 and the Debuncher respectively. The bottom half are the chromatic functions as defined in [6].

\begin{tabular}{lrrrr} 
& \multicolumn{2}{c}{ AP2 } & \multicolumn{2}{c}{ Debuncher } \\
& \multicolumn{1}{c}{ x } & \multicolumn{1}{c}{$\mathrm{y}$} & \multicolumn{1}{c}{$\mathrm{x}$} & \multicolumn{1}{c}{$\mathrm{y}$} \\
\hline \hline$\beta(\mathrm{m})$ & 7.127 & 10.398 & 4.940 & 14.964 \\
$\alpha$ & 0.691 & -1.644 & 0.384 & -1.951 \\
$\eta(\mathrm{m})$ & 0.014 & -0.03 & 0.010 & 0.0 \\
$\eta^{\prime}$ & -0.002 & -0.005 & 0.002 & 0.0 \\
\hline$W$ & 10.572 & 29.724 & 1.118 & 3.458 \\
$\Phi$ & 8.564 & 8.511 & 0.091 & -0.227 \\
$D \eta(\mathrm{m})$ & 7.596 & 3.034 & -0.633 & 0.0 \\
$D \eta^{\prime}$ & -0.824 & -0.104 & -0.028 & 0.0 \\
\hline
\end{tabular}

could have implications for the aperture in the Debuncher. We have not yet looked at apertures in the Debuncher to see if there are any problems due to the $\beta$-beat.

Even if it does not cause aperture restrictions this mismatch is fairly large and should be fixed as soon as possible. As soon as we have reconciled all the differences in the lattice files we will rematch it.

\subsection{Random Errors}

The following errors were considered: Alignment errors (transverse and longitudinal; roll, yaw and pitch) and field strength errors. Multipole errors have not yet been considered. Table 2 lists the errors that were used on all dipole and quadrupole magnets for most of the studies which are also the estimates for the real errors.

Table 2: Assumed RMS errors.

\begin{tabular}{lr} 
error & size \\
\hline \hline transverse alignment $(\mathrm{mm})$ & 0.5 \\
longitudinal alignment $(\mathrm{mm})$ & 0.5 \\
roll, pitch, yaw (mrad) & 10 \\
strength error & $1 \%$ \\
\hline
\end{tabular}

Using MAD [6], ten different seeds of machine errors were studied. At first, no attempt was made to correct the orbit (or anything else). 
The uncorrected orbits are typically in the range of several $\mathrm{cm}$ (peak excursion). So the orbit in the real beam line can be assumed to be significantly better than that. The resulting $\beta$-beat can be as high as $50 \%$.

The biggest problem caused by the machine errors is residual vertical dispersion due to vertical alignment errors of the quadrupole magnets. Dispersion values of more than $0.5 \mathrm{~m}$ have been observed in a number of seeds. The dispersion is a possible source of aperture restrictions as the nominal energy spread of the beam is $\pm 2.25 \%$ and the energy distribution is (to first order) flat. In addition, in most seeds the dispersion pattern is such that one has a particularly large vertical dispersion in the injection kicker which has the smallest vertical aperture (see Fig. 2 on page 4; the kicker is the last element in the line). Assuming nominal $\beta$-functions, a vertical dispersion of about $0.4 \mathrm{~m}$ in the kicker leads to scraping. If the $\beta$-beat is such that the $\beta$-function at that place is larger than nominal or there is a position offset, even a smaller dispersion would already lead to scraping.

In all the seeds, at least some scraping occurred somewhere in the line. All but one seed gave scraping in the injection kicker, mainly due to large vertical dispersion in that area. A small number of seeds also had aperture problems in and around the septum. However one has to keep in mind that this is for the uncorrected case so in the real machine the orbit excursions will be smaller. Nevertheless, the residual orbit excursions could still produce a significant amount of dispersion at some places, as local orbit bumps are not necessarily closed in dispersion. Usually, large local orbit bumps are rare in transport lines, however this line has a small number of corrector magnets that tend to be at similar phase advances, so it is possible to inadvertently introduce a local orbit bump of some amplitude while trying to optimize the line. The occurrence of $\pi$-bumps has been observed in simulating the orbit correction of the beam line, so it cannot be excluded for the real beam line.

\subsection{Vertical Misalignment of the First Two Doublets}

After looking in more detail at some of the seeds discussed above, it was noted that the one with the largest vertical dispersion has significant vertical alignment errors in the first two quadrupole magnets. We therefore studied the influence of misaligning individual quadrupole magnets, or pairs of them, on the vertical dispersion.

As the first four quadrupole magnets have hardly any vertical phase advance with respect to each other (see Table 3 on the following page) and have similar strengths, the effects of a vertical misalignment are very 
Table 3: Phase advances of the first four quadrupole magnets and the first three corrector magnets (from the start of the line, in units of $2 \pi$ ).

\begin{tabular}{ccc} 
element name & \multicolumn{2}{c}{ phase advance } \\
& horizontal & vertical \\
\hline \hline Q701 & 0.22 & 0.22 \\
Q702 & 0.23 & 0.22 \\
Q703 & 0.27 & 0.23 \\
Q704 & 0.28 & 0.23 \\
\hline PMAG & 0.06 & \\
KD.VT702 & & 0.22 \\
KD.HT704 & 0.30 & \\
KD.VT704 & & 0.35 \\
KH704 & 0.56 & \\
\hline
\end{tabular}

similar for each of those quadrupole magnets (modulo sign because there are two focusing and two defocusing quadrupole magnets) as is shown in Fig. 3 on the next page and Fig. 4 on page 10. They have a phase advance such that any vertical dispersion created due to a misalignment results in a large vertical dispersion in the kicker at the end of the line (see Fig. 4). Due to the length of the line, the vertical dispersion can grow to significant values if it is not corrected. Even if the orbit distortion caused by the misalignment is corrected, that does not mean that the dispersion is corrected, too.

To get a sense for the vertical alignment errors in this area of the real machine, one can look at the strength of the first vertical corrector magnet. As this magnet is essentially at the same phase advance as the quadrupole magnets (see Table 3), it should mainly cancel whatever kick results from misalignments of the first two doublets.

The vertical corrector near quadrupole 702 kicks the beam by about $0.28 \mathrm{mrad}$ [2]. If one misaligns one of the first four quadrupole magnets by about $0.1 \mathrm{~mm}$ one gets a kick of similar size at a similar phase advance. As all four quadrupole magnets have similar phase advances, from this, one can estimate that the combined misalignment of the four quadrupole magnets is about that value, i.e., small considering the overall alignment of the line.

The alignment of the first six quadrupole magnets has recently been checked. The effects of the measured horizontal and vertical misalignment on the lattice functions are shown in Fig. 5 on page 11. The upper graph 


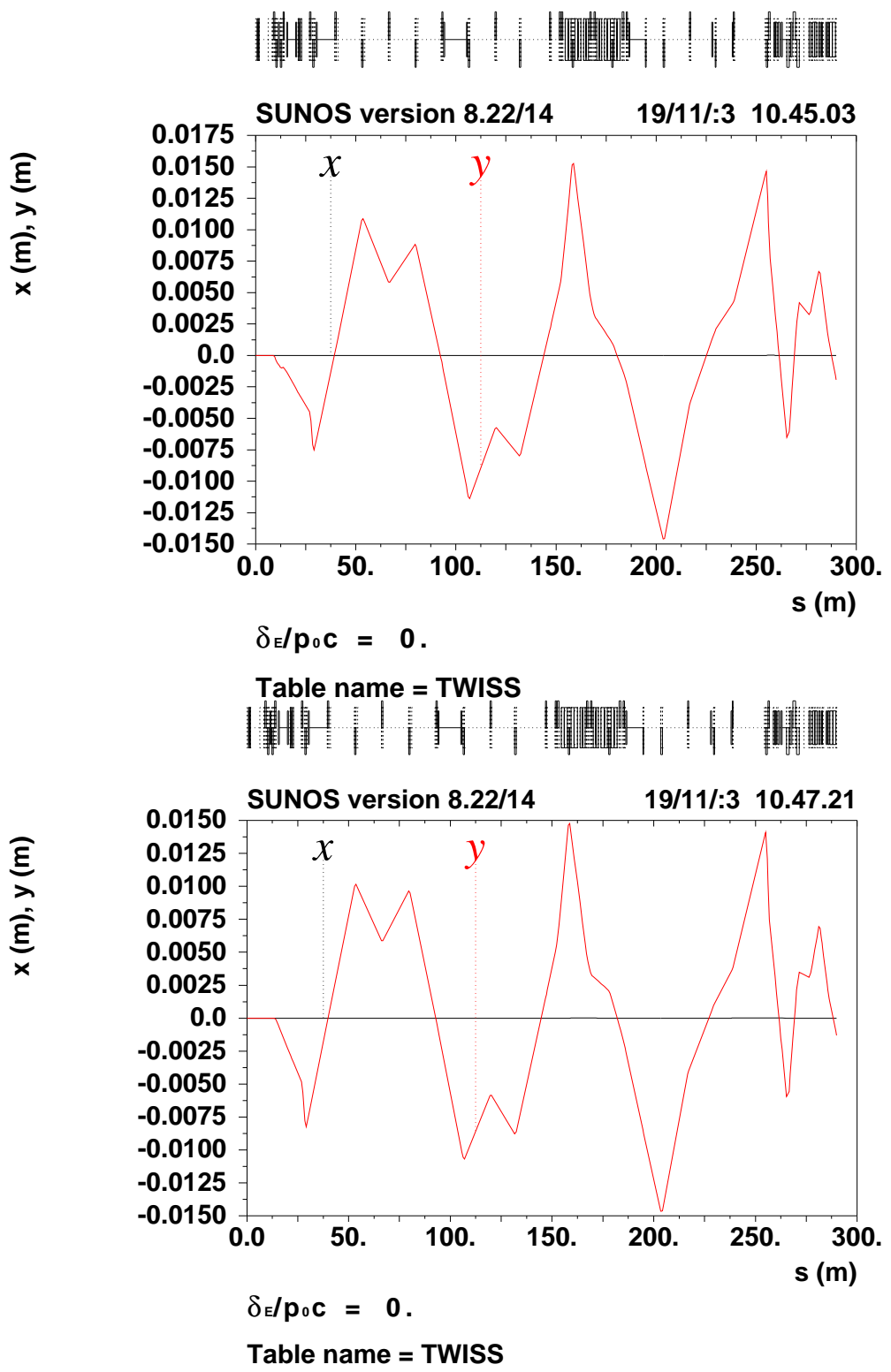

Figure 3: Orbit calculated assuming a $1 \mathrm{~mm}$ vertical alignment error for Q701 (top) and Q704 (bottom) (calculated using MAD [6]). 


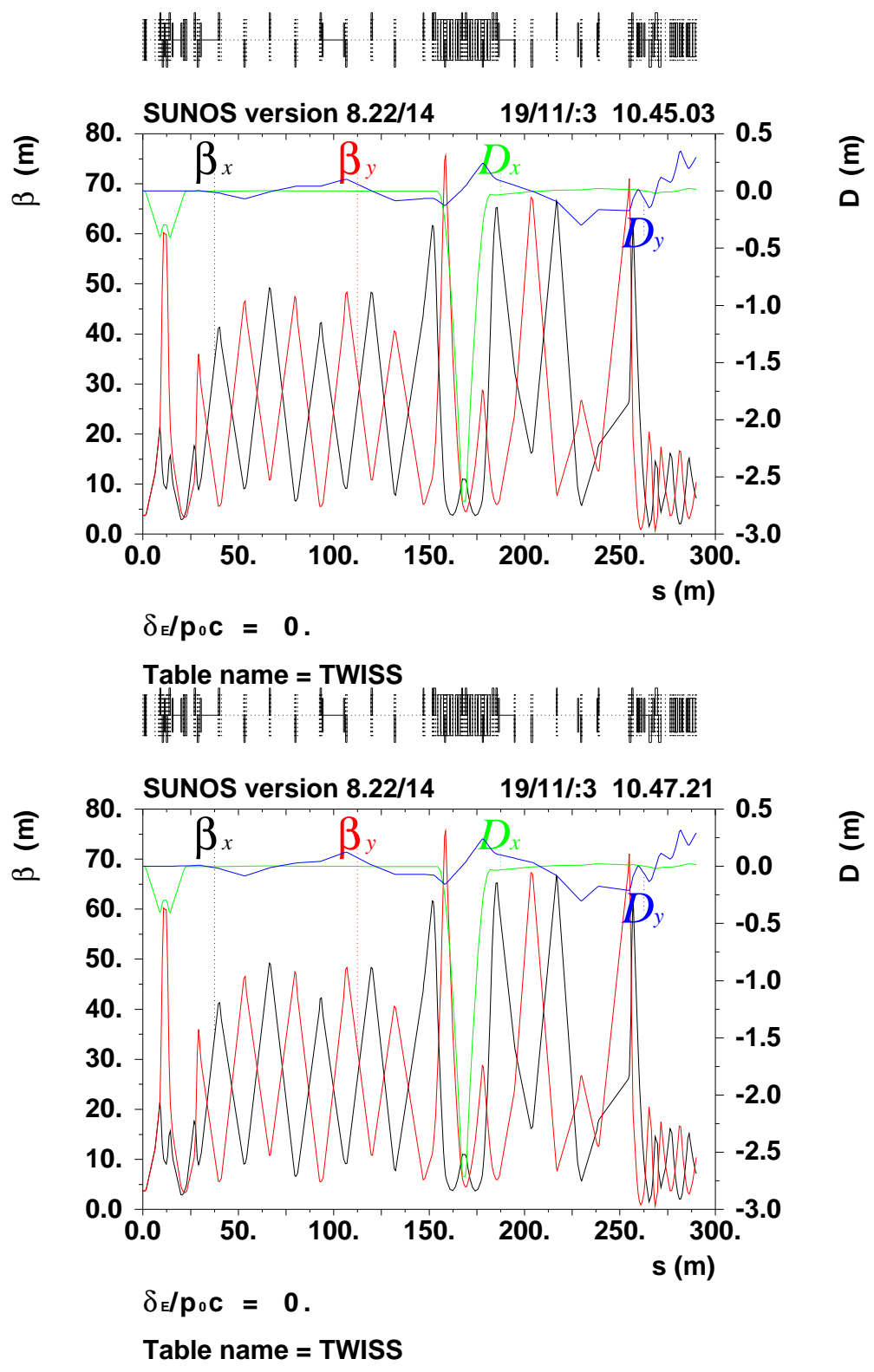

Figure 4: Lattice functions calculated assuming a $1 \mathrm{~mm}$ vertical alignment error for Q701 (top) and Q704 (bottom) (calculated using MAD [6]). 
in Fig. 6 on the next page shows the resulting orbit. Measured angle errors (yaw, pitch and roll) have not yet been included but are small enough that we assume that they do not contribute significantly. The peak excursions are of the order of a few $\mathrm{mm}$, which is small enough not to be a cause of worry. As the horizontal alignment is only with respect to some arbitrary line, we used deviations with respect to the average location of the magnets. This is potentially an optimistic assumption, however.

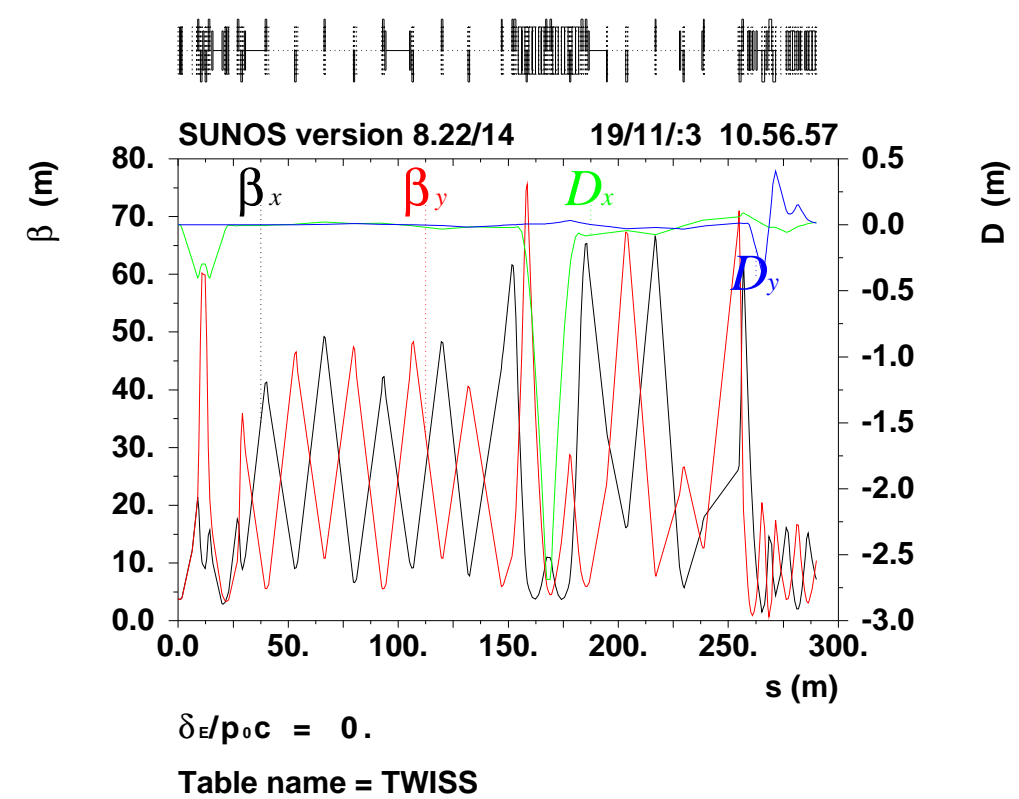

Figure 5: Lattice functions calculated using the measured transverse misalignment (calculated using MAD [6]).

The bottom plot in Fig. 6 on the following page shows the orbit from the misalignments combined with the first three vertical and one horizontal corrector strengths. The horizontal corrector is very weak so it does not change the excursion significantly. In the vertical plane the excursions downstream are now somewhat larger pointing to a significant error further downstream which is corrected using one or more of the first correctors, as there are not many vertical correctors after the first three until one gets past the momentum collimation area (see Table 4 on page 15). 


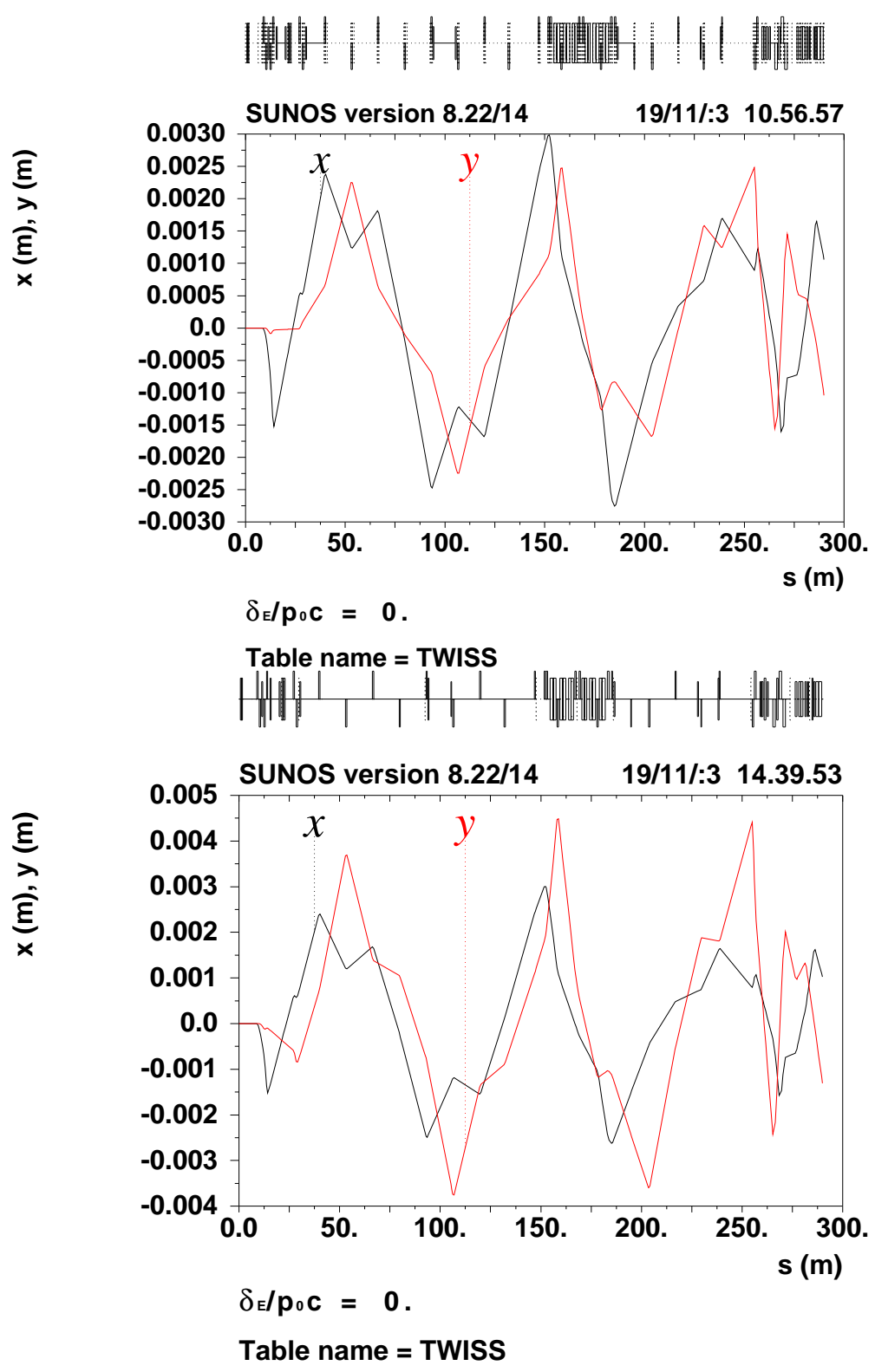

Figure 6: Orbit calculated using the measured misalignments of the first six quadrupole magnets without correction (top) and with the actual settings for the first three vertical and one horizontal corrector magnets (bottom) (calculated using MAD [6]). Note the different scale. 


\subsection{Orbit Correction}

In order to get an idea how well one can correct the orbit in the line, two typical seeds of machine errors were used. For each seed, the orbit was corrected with the MICADO-algorithm using the SEMs as orbit monitors ${ }^{3}$ and all corrector magnets and bending magnets as correctors. The monitors were assumed to be perfect, i.e., not misaligned and no readout errors.

These studies showed that, in the horizontal plane, one got large local orbit bumps at two places in the line even if the excursion was very small in that area to begin with (see Fig. 7). This triggered a more detailed study of the phase advances of correctors and monitors.

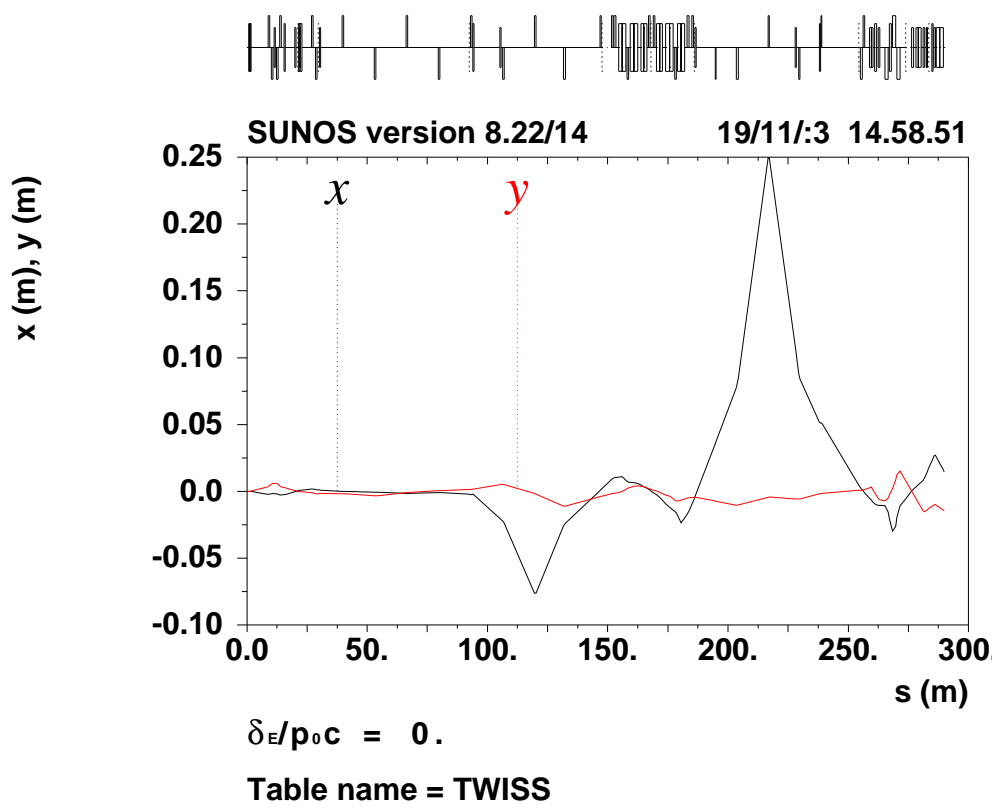

Figure 7: Orbit for a machine with random errors after one correction using MICADO (calculated using MAD [6]). SEM grids are denoted by a dashed line in the layout sketch. Note that both large horizontal bumps are invisible to the SEMs.

Table 4 on page 15 shows the phase advances between the orbit correctors and the SEM-grids that are used to measure the orbit. One can see

\footnotetext{
${ }^{3}$ The line is also equipped with about 30 beam position monitors, however for technical reasons so far they only work for forward protons, i.e. protons traveling along the beamline in the same direction as the anti-protons, which are hardly ever available. Therefore they are not considered for this study. There is currently work being done to make them available for reverse protons, i.e. protons from the Debuncher traveling in the opposite direction as the anti-protons [2].
} 
that a number of phase advances are either multiples of $\pi$ or $2 \pi$. In general, for a total phase advance of four (times $2 \pi$ ) one would like to have at least 16 correctors and monitors in each plane to cover all possible phase advances of errors. As here one has significantly fewer correctors and monitors, and in addition long stretches where certain phase advances are not covered at all, orbit correction is very difficult in the present line. This is aggravated by some correctors being at the same phase as a monitor instead of $\pi / 2$ downstream which is the optimum location for fixing the offset observed at the monitor. Also there are correctors with a phase advance of $\pi$ and $2 \pi$ with no monitor in between them. This makes possible $\pi$-bumps invisible to the monitors.

Correcting the orbit would be easier by adding and/or moving correctors and monitors. It is currently under study how many additional elements would be needed and where they should be located.

Another possibility is to rematch the lattice with a lower total phase advance. This should help with chromatic effects (discussed in Section 2.9) as well as changing the phase advance between correctors and monitors. This option is now being studied. Overall this should weaken the quadrupole strengths so there should not be an issue with either power converters being at their limit or magnets going into saturation. It would increase the $\beta$-functions on average but in most areas there is sufficient aperture to allow for that (see Fig. 2 on page 4).

\subsection{Measured Corrector Strengths}

Studying the settings of the corrector magnets as they are in the machine can serve two purposes:

1. If one of them gives a large kick to the beam this could indicate a large misalignment of a quadrupole magnet or some other large machine error.

2. Calculating the orbit using the ideal machine and the measured corrector strengths gives an indication of what the uncorrected orbit would look like. This can then be compared to the orbits generated using random seeds.

None of the correctors is at a very strong setting, so there is probably no gross error anywhere in the line. Figure 8 on page 16 shows the orbit one gets when putting the corrector strengths in. The rms value is smaller than the numbers observed when using the errors given in Table 2 on page 6 
Table 4: Phase advances from the start of the line to the correctors and monitors (in units of $2 \pi$ ). Values printed bold mark correctors with similar phase advance, lines connect monitor-corrector pairs at the same or $\pi$ phase advance.

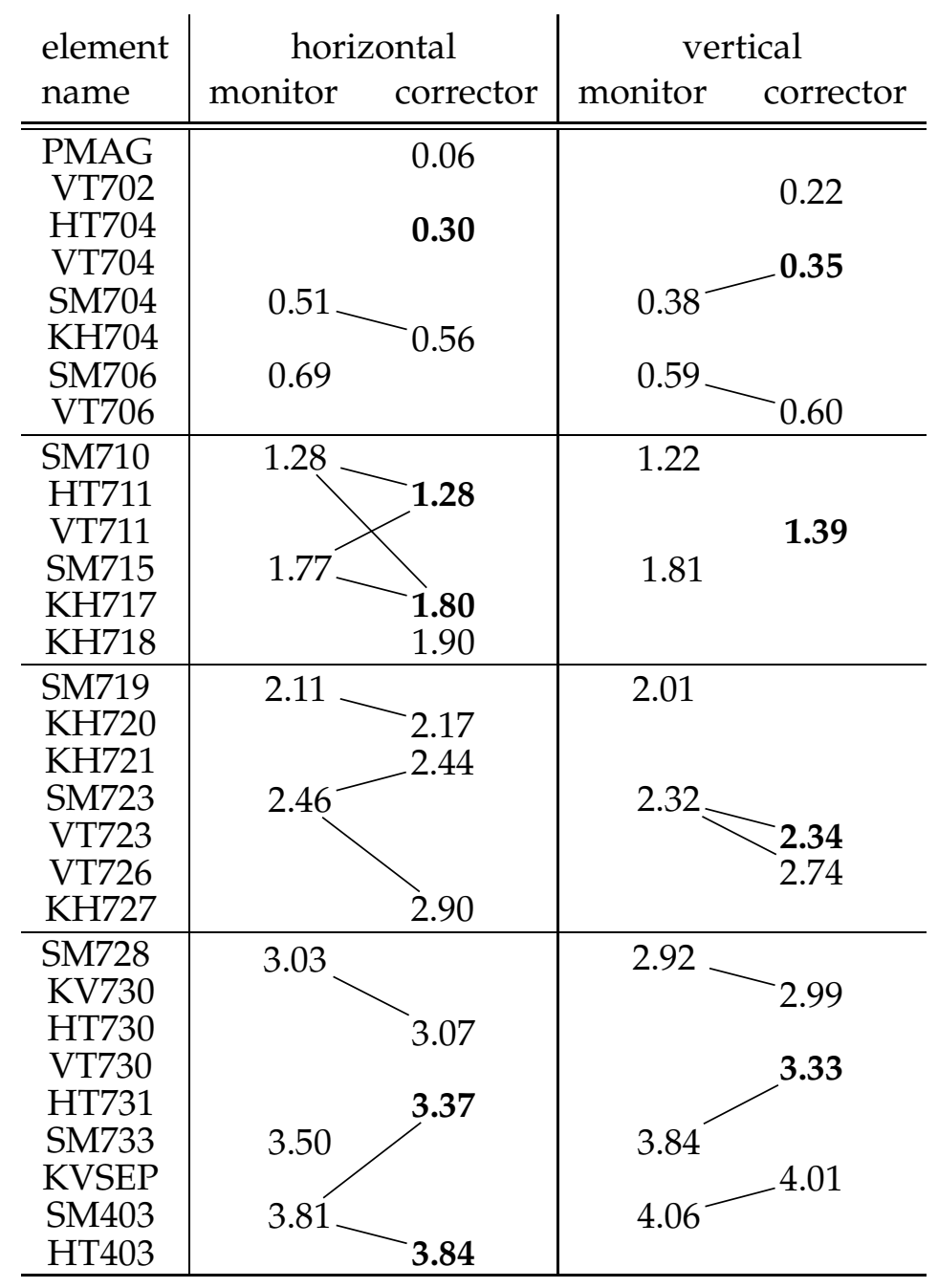


and a number of random seeds. This is either due to the real errors being smaller than estimated or due to the fact that there are only very few correctors available which in addition do not cover the different phase advances very well (see Table 4 on the page before).

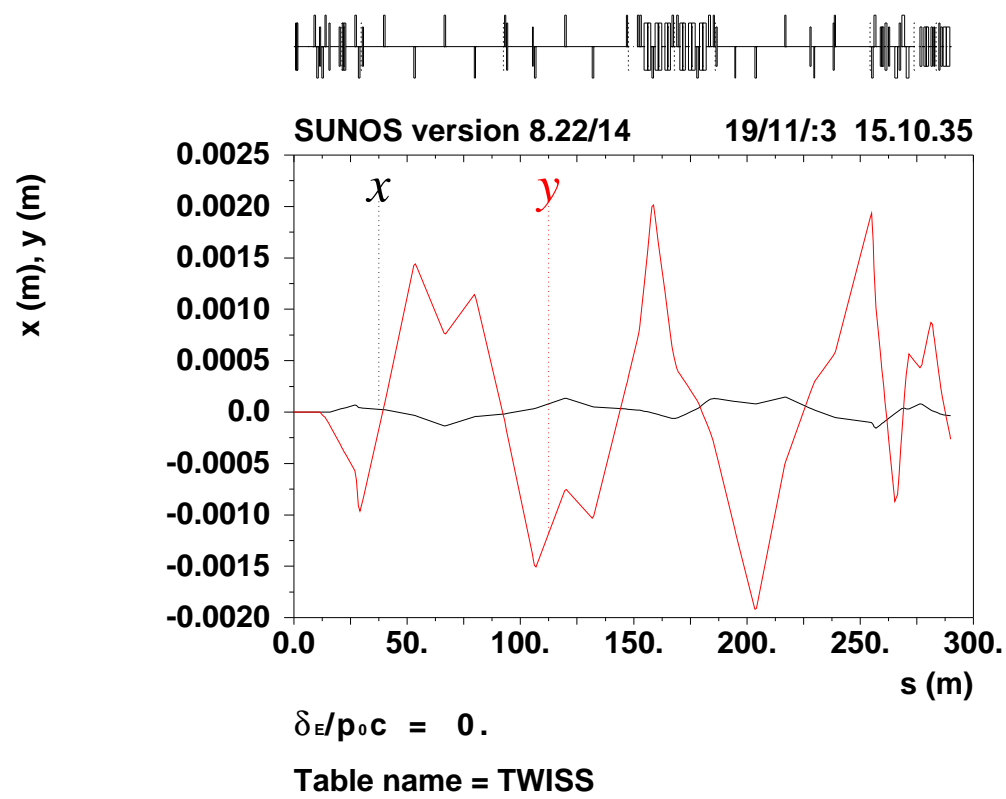

Figure 8: Orbit calculated using the real settings of the corrector magnets (calculated using MAD [6]).

\subsection{Field Errors in the Injection Channel}

The beam goes off center through the first three quadrupole magnets in the Debuncher (Q405 to Q403). Especially in Q405 the offset is very large so that it is not clear that one can still assume a perfect quadrupole field at nominal strength. This is currently being studied.

\subsection{Off-Momentum Lattice}

Figure 9 on the facing page shows the chromatic $\beta$-functions as defined in [6]. They were calculated assuming an initial value of zero at the start of the line. As the beam coming out of the target is fairly uniform we assume this to be at least a reasonable guess as it is not straightforward to give a better estimate. 

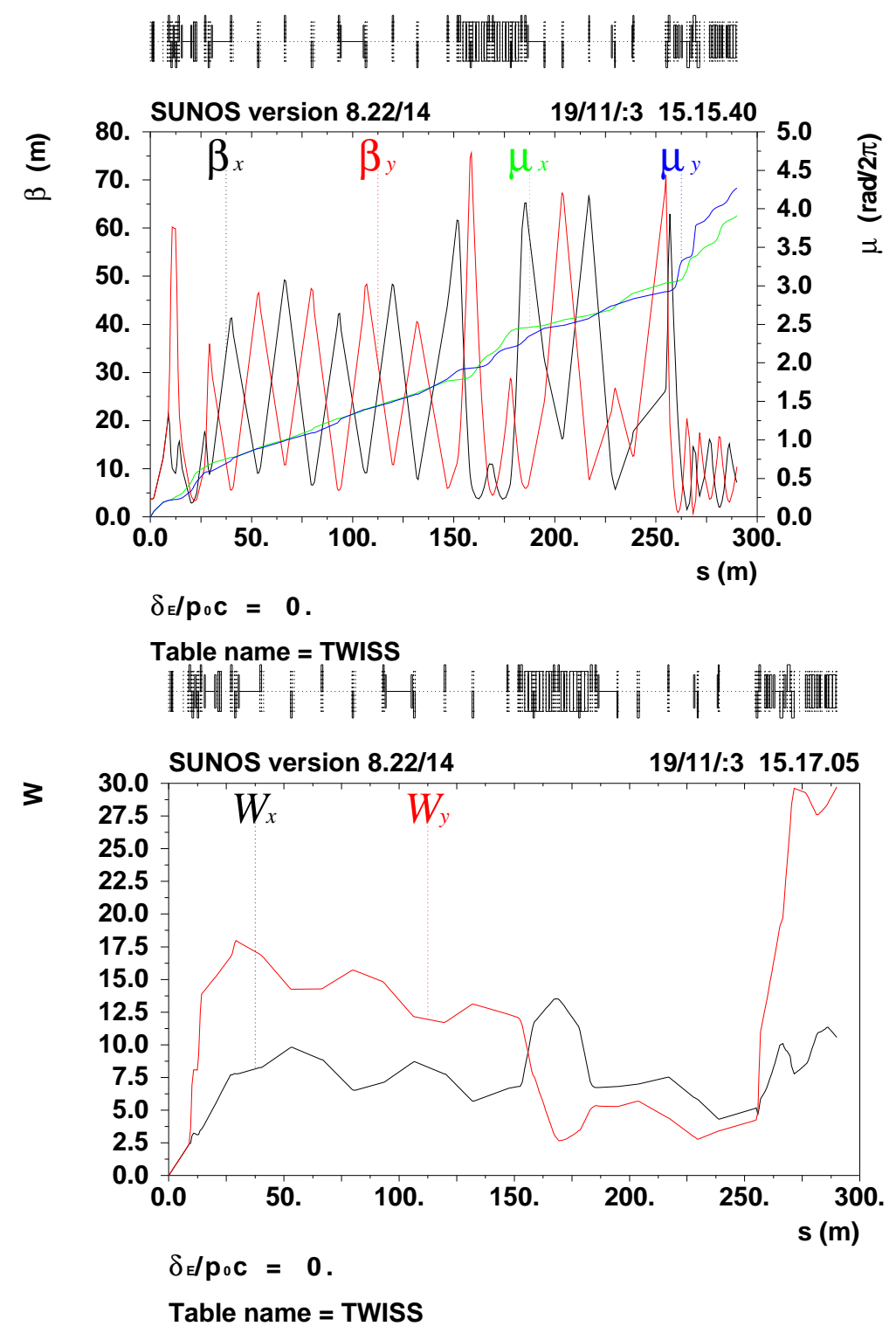

Figure 9: $\beta$-functions and phase advances (top) and chromatic $\beta$-functions (bottom) of the AP2 beam line calculated with MAD [6]. 
The chromatic $\beta$-functions are rather large, especially at the end of the line, which means that they are not matched well into the Debuncher ring (as rings tend to have fairly small chromatic $\beta$-functions; see Table 1 on page 6). This is also visible looking at the $\beta$-functions for particles with a momentum deviation of $2 \%$, which show a $\beta$-beat of the order of $50 \%$. As the energy distribution in the beam is flat (to first order) this leads to a large mismatch for a significant fraction of beam particles.

In general, experience with other beam lines has shown that the chromatic $\beta$-functions should be smaller than 5 [8]. Experience from other machines $[8,9]$ has also shown that the chromatic lattice functions need to be matched at both ends of a transfer line for beams with a significant energy spread. There are two possibilities to do that for this line:

1. If the chromatic functions at both ends of the line are known (at the end of the line they are easily obtainable from the lattice of the Debuncher, the upstream end might be more difficult), one can simply rematch the line to be matched not just on-energy but also in terms of the chromatic functions.

2. If the upstream values are not obtainable one could try to develop four orthogonal knobs (two for each plane) which change the chromatic functions at the beginning of the line but leave them constant at the end of the line. With the knobs it should then be possible to empirically match the line.

It remains to be demonstrated that magnets at suitable locations are available for this purpose. In addition, both possibilities might require sextupole magnets and possibly even higher order multipoles currently not available in the line.

The necessity of better matching and reducing the chromatic functions is also obvious from the tracking results, which indicate that off-momentum particles "see" a significantly different lattice and therefore different tight spots compared to on-momentum particles. The fairly large chromaticity ( -10 in both planes) also indicates possible problems for off-momentum particles. This problem might be alleviated by rematching the line for a lower total phase advance and chromaticity, but it might also require sextupole magnets. Rematching the lattice is currently under way. 


\section{Tracking Studies}

Tracking studies were done to better understand the behavior of off-momentum particles and to understand the loss mechanism. Tracking was done either with a grid of particles filling the aperture or with particles generated with MARS, which simulates the production of anti-protons in the target and should therefore correspond to the real distribution of particles in the beam.

\subsection{Tracking a Grid of Particles}

To get a first impression of what happens to particles with a large amplitude and/or a large momentum deviation, a grid of particles was tracked through AP2 starting after the lithium lens and ending after the injection kicker. Apertures corresponding to the ones shown in Fig. 2 on page 4 were included in the tracking of the line to model losses properly.

All on-momentum particles inside the aperture of $40 \pi \mathrm{mm}$ mrad survive. Figure 10 shows the final coordinates of a grid of particles filling the whole aperture. All particles are on-momentum and were launched with only transverse offsets but no angles.

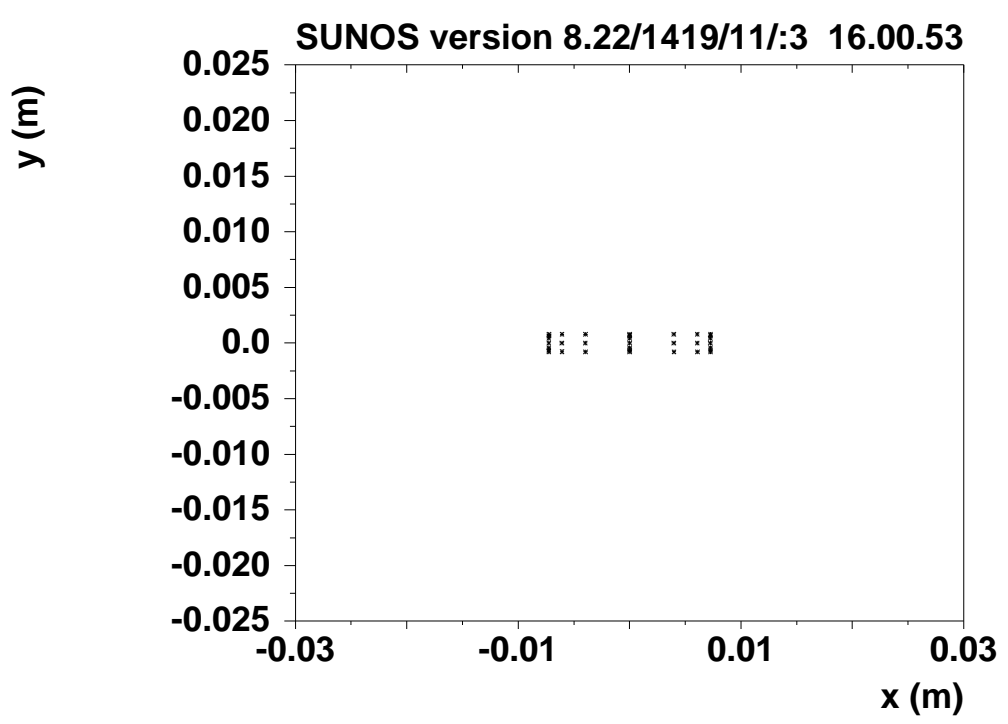

Table name $=$ TRACKING

Figure 10: Final transverse coordinates (after the injection kicker) of a grid of onmomentum particles tracked through AP2 (calculated using MAD [6]). 
Figure 11 on the facing page shows the same plots for particles with a momentum deviation of $\pm 2.25 \%$. One can see that a number of particles are lost and that the particles end up at very different transverse positions compared to the on-momentum particles, although they were launched at the same initial transverse positions. This is a strong indication that the lattice is very different for off-momentum particles compared to onmomentum particles.

The losses occur through different mechanisms in the two cases shown here. For $\frac{\Delta p}{p}=-2.25 \%$, the losses occur in the horizontal plane and mainly for particles with large positive amplitudes but not for those with large negative amplitudes. Also, most particles lost horizontally are lost in the high dispersion area. This indicates that those particles have a larger than nominal $\beta$-function in the momentum collimation area and therefore that one side of the beam is scraped off preferentially. For $\frac{\Delta p}{p}=+2.25 \%$, the losses occur in the vertical plane and mainly on the side with positive vertical amplitudes. The vertical losses in general are distributed along the line more evenly than the horizontal losses.

Figure 12 on page 22 shows how the final transverse amplitudes depend on the momentum deviation. One can see that the scaling is not linear, indicating non-linear behavior of the chromatic functions in the relevant energy range.

\subsection{Tracking a Set of Particles Generated by MARS}

Figure 13 on page 23 shows the normalized amplitude and longitudinal distributions of particles generated with MARS for all particles and for those that are transmitted through AP2. There is an aperture restriction in the vertical plane corresponding to about $32 \pi \mathrm{mm}$ mrad. Most lost particles that have a small horizontal amplitude have a large vertical amplitude and vice versa. Most losses occur either if a particle is at an initial amplitude of more than $40 \pi \mathrm{mm}$ mrad in one plane or for particles with a large momentum deviation. Particles with an amplitude of more than $40 \pi \mathrm{mm} \mathrm{mrad}$ would be lost in the Debuncher anyway. However it is worrying that a significant number of particles with an amplitude smaller than $40 \pi \mathrm{mm} \mathrm{mrad}$ are lost, mainly because they have a large energy deviation. Those particles could be transmitted if the chromatic properties of the line were improved. Figure 14 on page 24 shows the same data set, only this time looking at particles with an initial amplitude of not more than $40 \pi \mathrm{mm}$ mrad as higher amplitude particles would be lost in the Debuncher anyway. The same set 

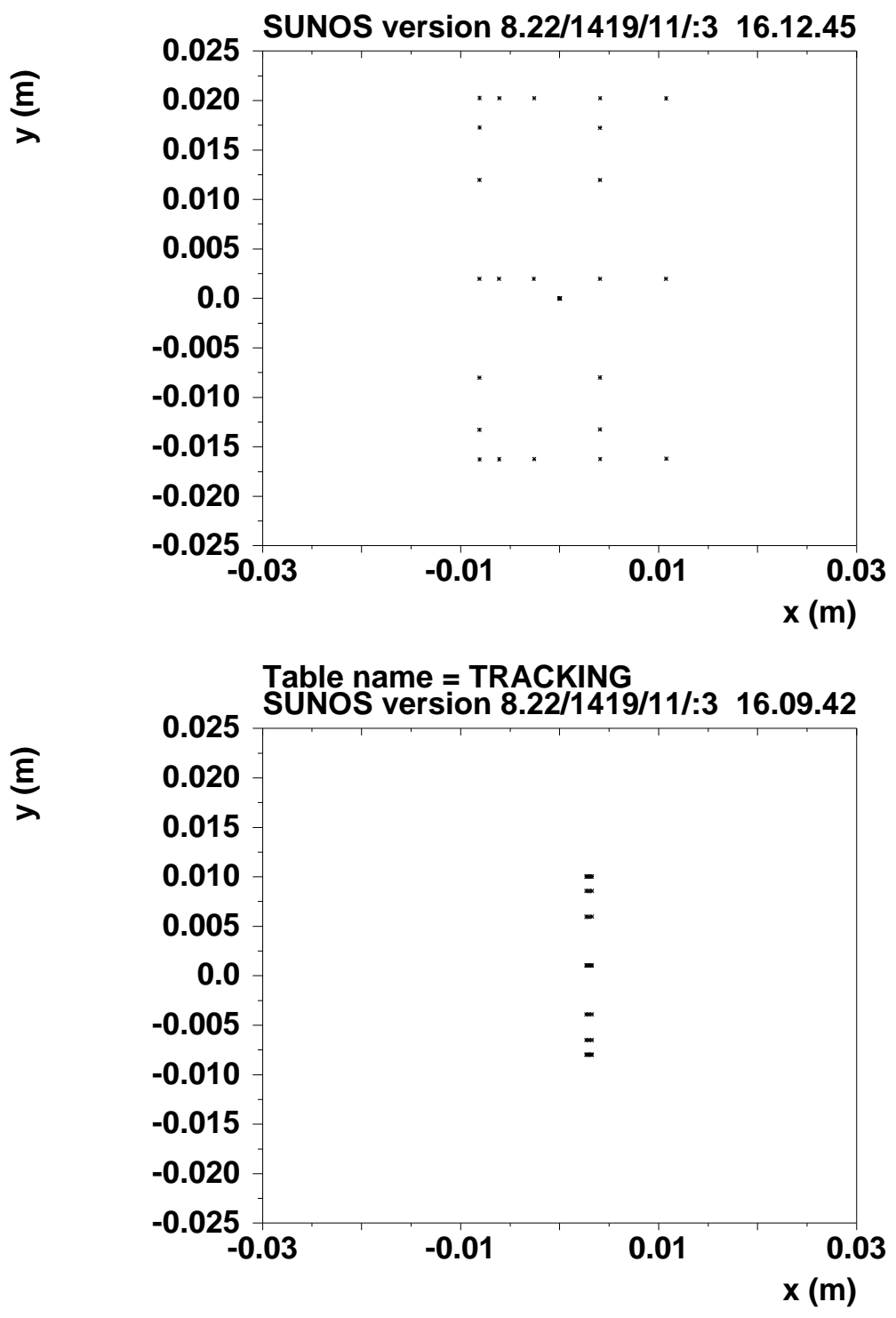

Table name $=$ TRACKING

Figure 11: Final transverse coordinates of two grids of off-momentum particles tracked through AP2 (calculated using MAD [6]). The particles in the top graph have $\Delta p / p=-2.25 \%$, in the bottom one they have $\Delta p / p=+2.25 \%$. The transverse launching positions are identical to the particles shown in Fig. 10 on page 19. 

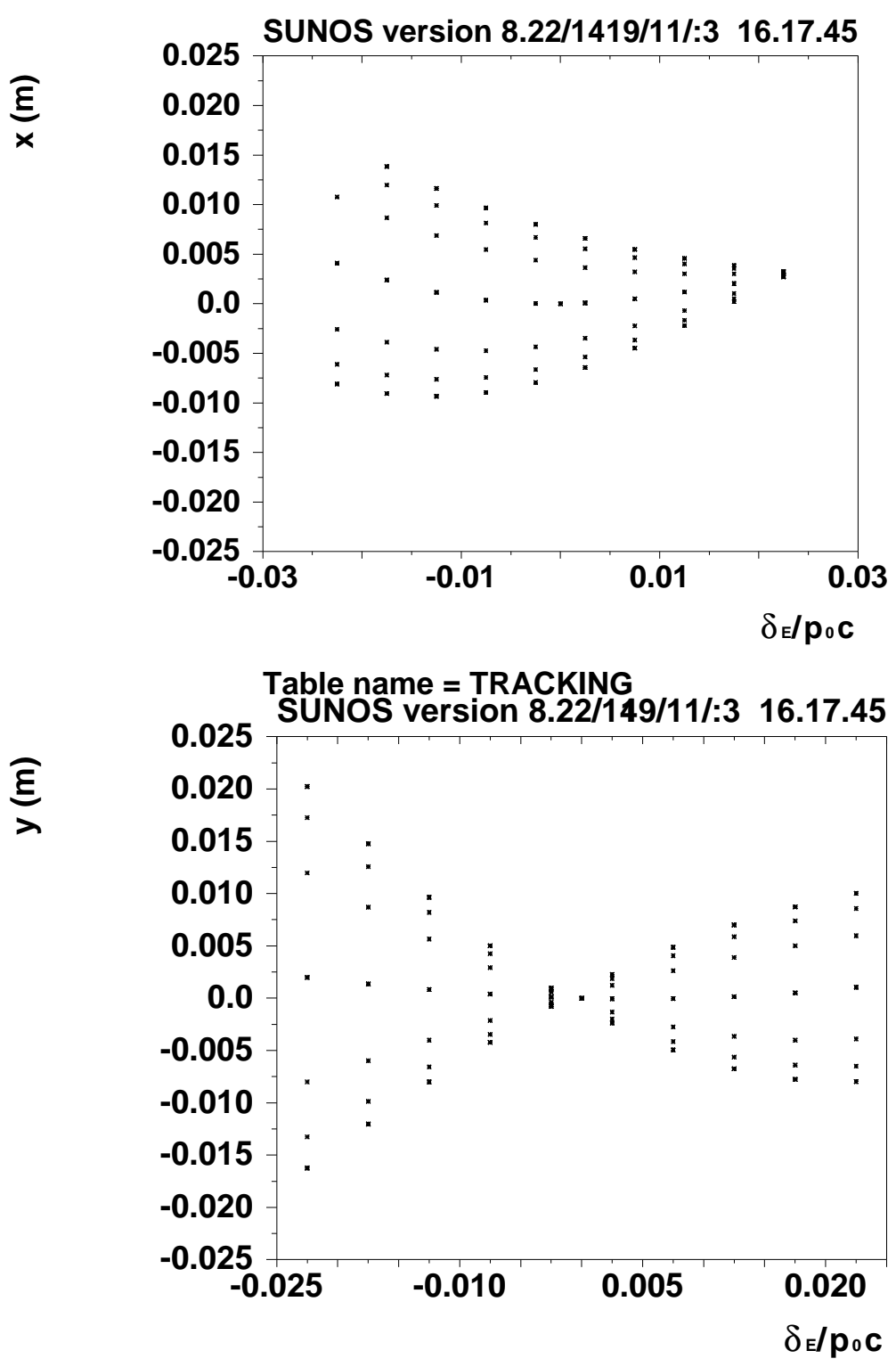

Table name $=$ TRACKING

Figure 12: Final coordinates of a grid of particles tracked through AP2 (calculated using MAD [6]). The top graph shows the dependence of the final horizontal position on the momentum deviation. The bottom graph shows the same for the vertical plane. All particles were launched with only a transverse offset and a momentum deviation, but no transverse angles. 

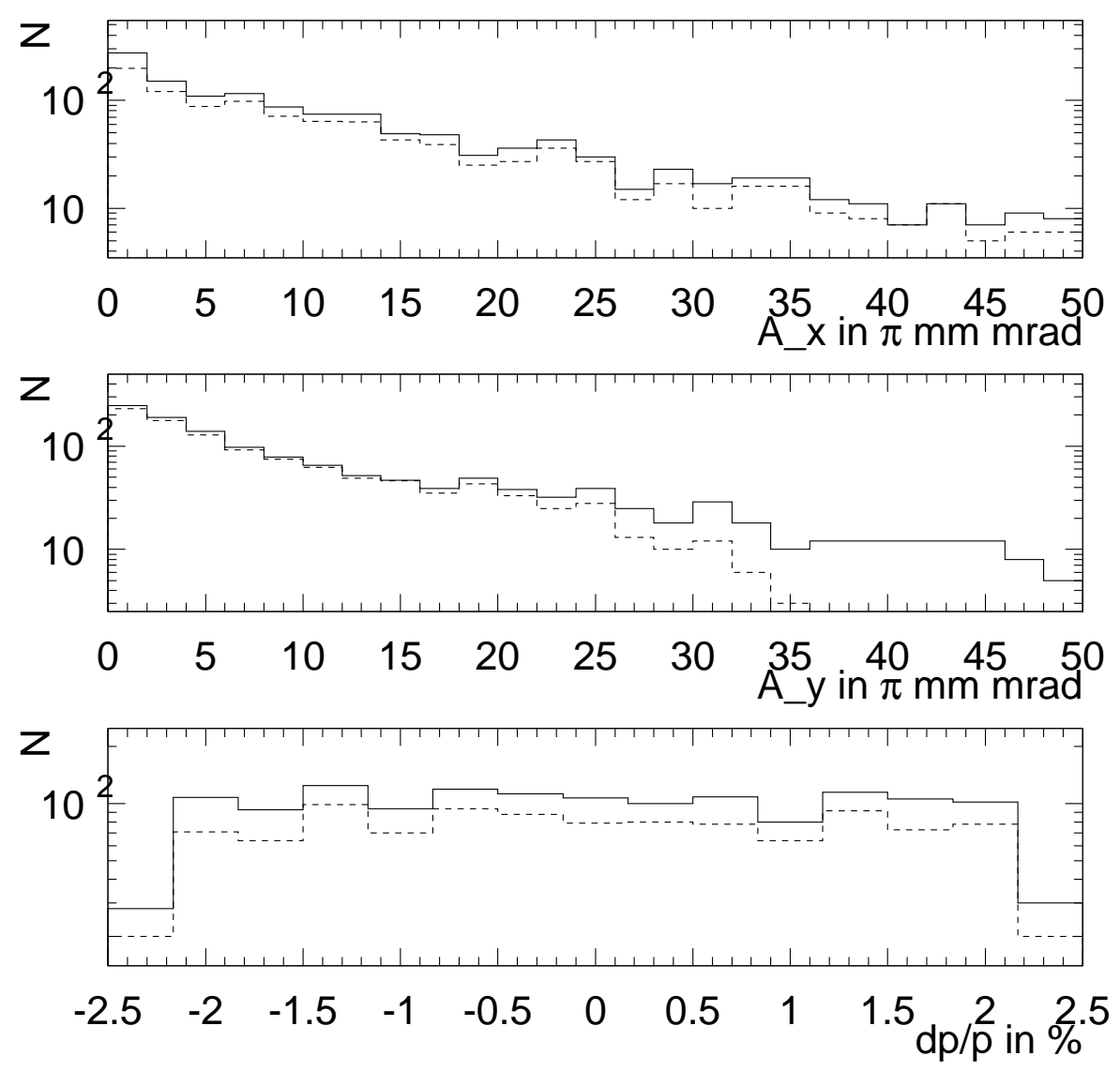

Figure 13: Initial distributions of particles generated with MARS. The solid line represents all particles, the dashed line particles that are transmitted through AP2 using the ideal lattice with collimation representing the vacuum chamber. 

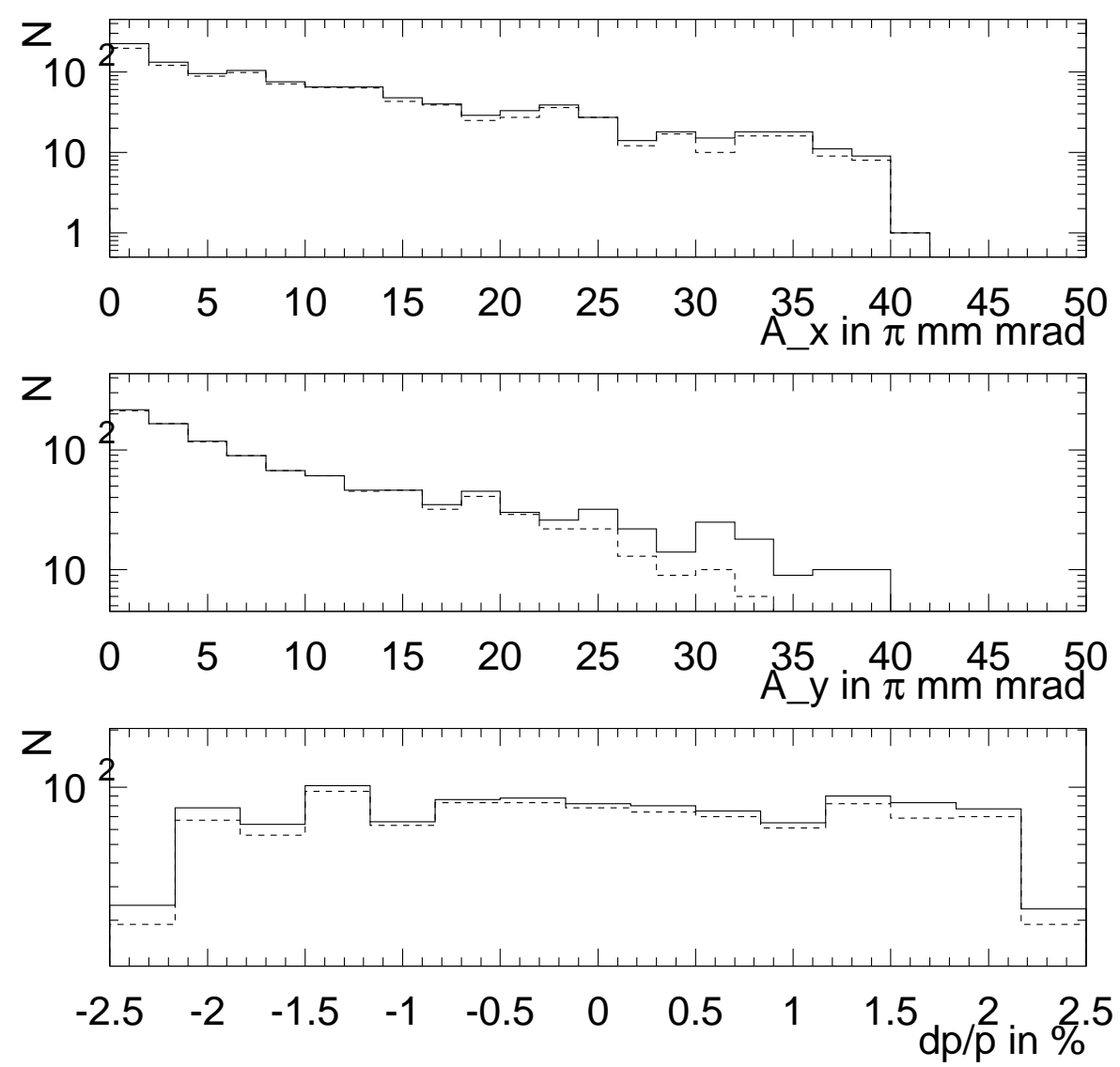

Figure 14: This is the same plot as in Fig. 13 on the page before but this time all particles with an initial amplitude of more than $40 \pi \mathrm{mm}$ mrad have been discarded. 
of particles was also tracked through a lattice with machine errors. The results are the same.

To study the effect of chromatic effects, the same set of particles was tracked with the energy deviation of all particles set to zero. In that case the transmission was increased by about $3 \%$. As this was done using the ideal lattice we assume the effect in the real machine to be larger.

As the line is not perfectly matched into the Debuncher (especially in terms of chromatic functions), the behavior of the normalized amplitude was also studied. The set of particles generated with MARS was tracked through the line and then injected into the Debuncher and tracked there for a number of turns. Currently there are no aperture restrictions in the Debuncher lattice in the simulation, so all particles will survive once they get into the Debuncher. Figure 15 on the following page shows the normalized amplitudes of the particles surviving the line at the start of the line, at the end of it, and after one, two and ten turns in the Debuncher. Figure 16 on the next page shows the projection of the distribution using the same color code.

One can see that in the vertical plane the amplitude of some particles increases significantly. The amplitudes change again turn by turn in the Debuncher but the changes there are rather small. However this needs to be studied in more detail once the positions and strengths of the injection

channel quadrupoles are sorted out and the apertures are included in the model of the Debuncher.

\section{Other Sources of Aperture Restrictions}

Aside from imperfections and chromatic effects there are other possible sources of acceptance restrictions.

\subsection{Energy Error in the Debuncher Ring}

Since the maximum momentum aperture in the line is $4.5 \%$ total width, a significant fraction of particles could be lost if the energy of the Debuncher were significantly different from the central energy transmitted by the AP2 line, as the energy acceptance of the Debuncher is $4.6 \%$.

The absolute energy acceptances of the Debuncher and the line have been measured [7]. They agree well. However this does not exclude the central frequency of the Debuncher being not in the center of the energy acceptance which would introduce a significant $\beta$-beat in the Debuncher. 


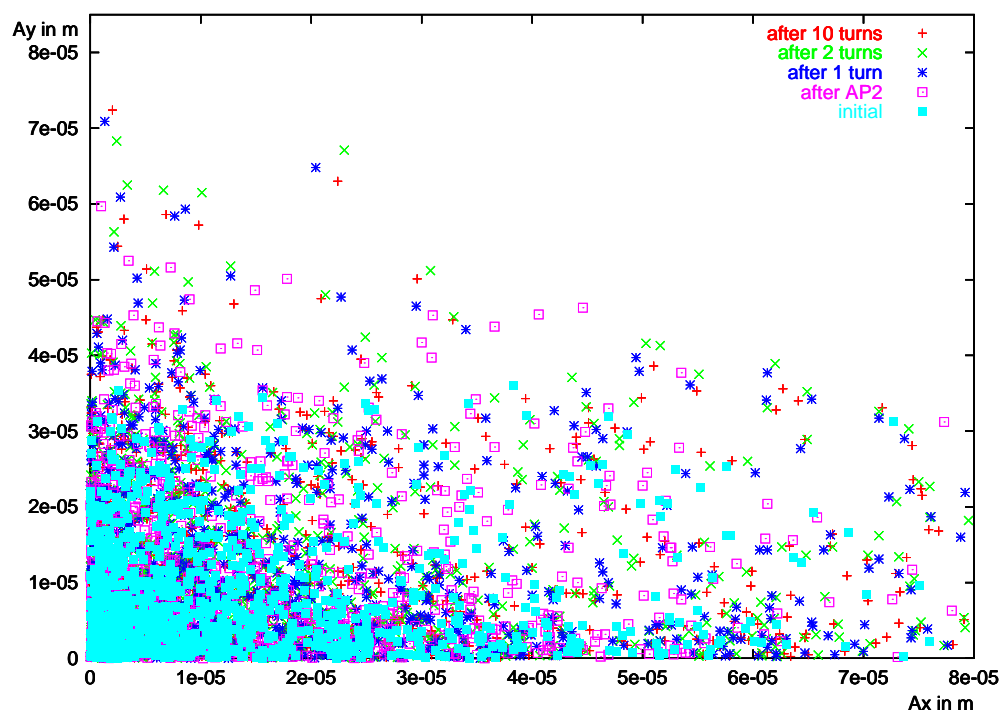

Figure 15: Particles from MARS. Normalized amplitudes at various places. Uses only surviving particles.
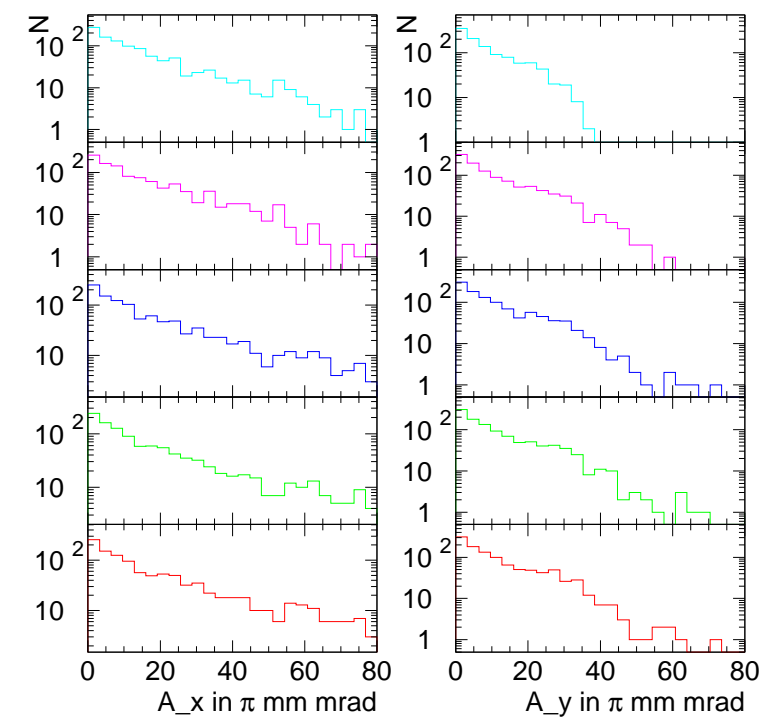

Figure 16: Projections of Fig. 15. From top to bottom: initial, after AP2, after one, two and ten turns in the Debuncher (same color code as in Fig. 15 is used). 


\subsection{Misalignment of a Small Aperture}

There is always the possibility that a small aperture like the kicker or the septum is misaligned and therefore restricting the acceptance of the line. This could be checked using closed orbit bumps to move the beam in the areas where the aperture is believed to be tightest, provided correctors with suitable phase advance are available. This has not been studied yet and might be difficult due to the scarcity of corrector magnets.

\subsection{Multipole Errors}

The effect of multipole errors has not yet been studied. They could make the lattice worse for off-momentum, large amplitude particles. Unfortunately, at the moment data on typical multipole errors for the magnets is not available for study [2]. This might be especially important for the Debuncher magnets through which the beam travels significantly off center in the injection channel (quadrupoles 403 to 405). We are currently working on incorporating the multipole fields of quadrupole 405 into the simulation as this is the magnet where the beam is off-center by more than $10 \mathrm{~cm}$.

\section{Suggested Experiments}

The suggested experiments serve to study if some of our suspected aperture restriction sources are actually a problem. Some of the experiments will simply prove or disprove a source whereas others will possibly fix the problem if it is present.

\subsection{Beam-Based Alignment}

Beam-based alignment offers a possibility to correct the orbit better than using the monitors and transmission alone. A well corrected orbit usually has a small dispersion, so any problems from dispersion in tight apertures might be reduced.

\subsection{Reducing Possible $\pi$-Bumps}

The possible $\pi$-bumps mentioned in Section 2.6 could be easily fixed by applying a $\pi$-bump until maximum transmission is obtained, although with the large energy spread combined with large chromatic lattice functions they would not be closed for all energies. This is a simple experiment which 
can be done parasitically and could possibly increase the transmission immediately.

Some first measurements have recently been done [7]. The results are not conclusive for some combinations as one could change the strength only by a small amount. For some combinations it looks like the beam is well centered in the aperture. One particular combination showed significant leakage. We are studying this as it might indicate a quadrupole with a significant strength error between the two correctors (although it could also be due to the fact that the phase advance is not exactly $\pi$ even in the model).

\subsection{Dispersion Bumps to Cancel Dispersion at the Kicker}

If there is a significant amount of vertical dispersion in the kicker, this could restrict the acceptance of the line. This could be checked by using two closed orbit bumps upstream of the kicker such that one of them creates dispersion at the kicker and the other one a slope of the dispersion at the kicker. One would then simply tune those bumps on transmission.

With the limited number of correctors in the line it is not straightforward to find to knobs that create appropriate dispersion waves that are $90^{\circ}$ out of phase, create a significant amount of vertical dispersion and at the same time do not change the orbit by too much. We found two knobs and their effects on the lattice and the orbit are shown in Fig. 17 on the facing page. However some of the correctors need to kick the beam by more than $1.5 \mathrm{mrad}$ for a modest amount of dispersion whereas the correctors can only provide a kick of 0.9 mrad [7]. It is therefore not clear if much can be gained from this study. As an experiment would be brief and could be done parasitically, we nevertheless recommend it. It will not be possible to cancel any large amount of dispersion, if it is present, but it could at least indicate its presence.

\subsection{Closed Orbit Bumps in Small Apertures}

If one of the small apertures, such as the kicker, is severely misaligned, this can be a strong aperture restriction. This can be checked by moving the beam in the smallest apertures using closed orbit bumps (again assuming suitable corrector magnets are available). Once the bumps have been calculated, this experiment can be done parasitically. 

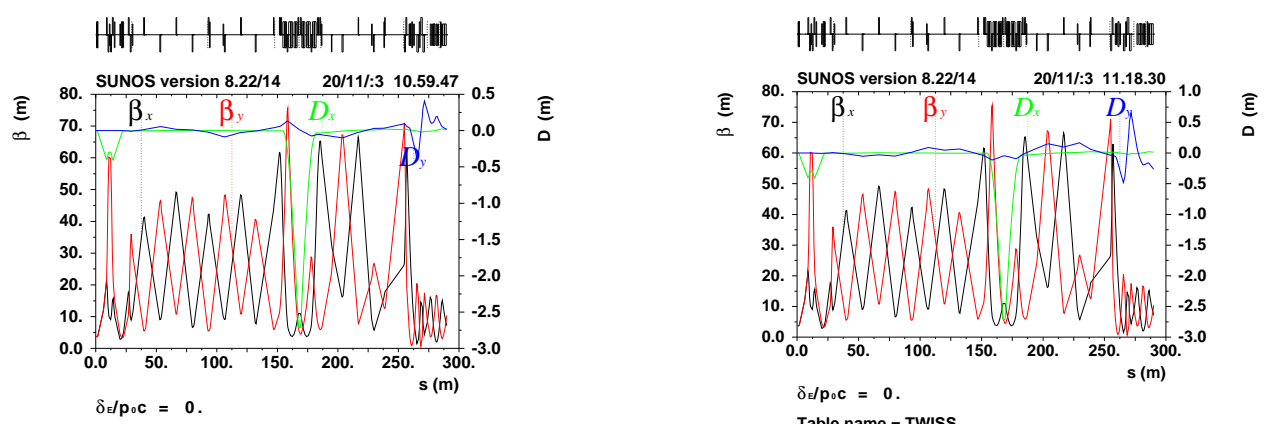

Table name $=$ TWISS
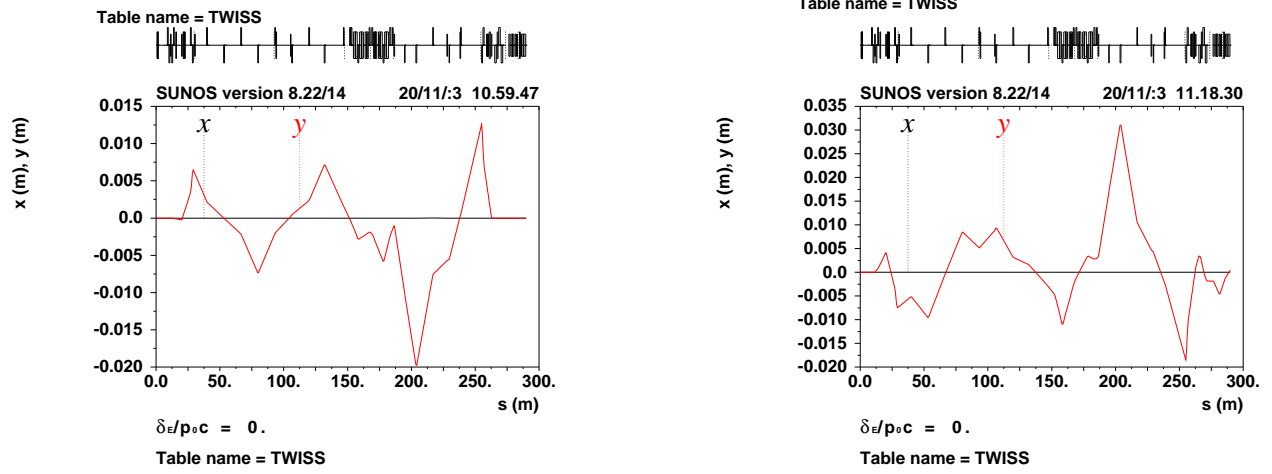

Figure 17: Lattice functions (top) and orbit (bottom) for two dispersion knobs (calculated with MAD [6]).

\subsection{Energy Error in the Debuncher}

As mentioned in Section 4.1, the central energy of the Debuncher could be off compared to the central energy of the beam line. We suggest the following experiments to study if this reduces the transmission:

The central frequency ${ }^{4}$ of the Debuncher should be measured. This needs to be done with protons, so it needs dedicated beam time. The measurement itself is fairly straightforward so it should not take too long. The results should be compared to the measured energy acceptance of the Debuncher and to the frequency at which it is actually operating.

If the central frequency is not in the center of the energy acceptance, the Debuncher should be adjusted such that it is centered. For house-keeping purposes it is recommended to measure the central frequency occasionally

\footnotetext{
${ }^{4}$ The central frequency is the revolution frequency at which the beam on average travels through the centers of the quadrupole magnets. It is usually measured by measuring the horizontal tune for a number of different revolution frequencies and sextupole magnet settings assuming that the centers of the sextupole magnets are on average the same as the centers of the quadrupole magnets.
} 
to make sure it does not change significantly.

\subsection{Momentum Slicing Using the Momentum Collimator}

This experiment serves to determine how the transmission depends on energy by selecting slices of energy with the collimator and measuring the resulting transmission. This should be done with and without bunch rotation in the Debuncher ring as this could influence the capture efficiency differently for different energies [7]. If there is a significant amount of residual dispersion at a tight place, this experiment should show reduced transmission for off-energy particles.

\subsection{Empirically Matching the Initial Lattice Functions}

In case there is a mismatch of the lithium lens, this could lead to a large $\beta$ beat, restricting the aperture. If this is indeed the case, this can be fixed in a straightforward manner by using knobs that change the initial lattice functions but do not change them at the end of the line. This experiment can be done parasitically as one would simply tune the settings of the matching knobs on transmission.

\subsection{Matching the Chromatic Lattice Functions}

Not only the on-energy lattice functions need to be matched, the off-energy ones should be matched as well.

If the values at both ends of the line are known, one can rematch the line. Setting up the rematched lattice probably requires dedicated machine time unless the changes are small and one can therefore "knob" from the old to the new settings.

If the values are only known at the end of the line, one can calculate knobs that change the chromatic lattice functions at the beginning of the line but leave them unchanged at the end. These knobs can then be used to tune the line. The tuning can be done parasitically. Note that currently the chromatic functions are not matched at the end of the line. This should be fixed first; depending on how large changes are required, this might necessitate dedicated beam time.

Any matching of the chromatic functions might require sextupole magnets or even higher-order multipoles, which are currently not in the line. It could be done in conjunction with a proposed lowering of the total phase 
advance of the line. The aim should also be not just to match the chromatic functions at both ends but to also lower them throughout the line.

\subsection{Measure and Correct Dispersion}

The obvious way to fix possible problems from dispersion would be to simply measure and correct it. However this is not that easy.

It is more or less impossible to measure the dispersion using the antiproton beam. The only chance one might have to measure it in the area where it is most important (the septum and kicker, as those are the smallest apertures) is to do momentum slicing and measure the orbit for the different momentum slices. But the intensity might be too low to get meaningful results.

It should be possible to measure dispersion using reverse protons. However, this will not be as helpful as it may seem as we are not looking for nominal dispersion but for residual dispersion caused by imperfections. So any dispersion caused by the first two doublets will only show up between the doublets and the lithium lens but not at the kicker, where we think it might cause a problem if it is of sufficient size. Currently, we think it is not worth the effort and machine time to try to measure the dispersion with reverse protons. However once the beam position monitors become available for reverse protons one could think about response matrix type measurements.

In case forward protons are available one should definitely attempt to measure the dispersion (and possibly correct it), as in that case the relevant thing would be measured.

\section{Recommendations}

The recommendations given here are roughly ordered such that those mentioned first should be implemented first because they are either very important or because it is very simple and cheap to implement them.

\subsection{Match Into Debuncher}

As it is currently not clear, how well the end of the line is matched into the Debuncher, this should be sorted out quickly. Once the strengths and positions of the magnets in the Debuncher are established, one should rematch the end of the line to make sure all lattice functions are matched. It might be worth developing knobs for empirical matching of the line. 


\subsection{Get BPMs to Work for Reverse Protons}

Diagnosing and steering the line should be much improved if one has BPMs available at almost every quadrupole for reverse protons. It will probably allow for response matrix type measurements which should help in finding sources of residual dispersion.

\subsection{Develop a Technique for Beam-Based Alignment}

Beam-based alignment should improve the orbit and the dispersion. Unfortunately it is not entirely obvious how to do it. Using anti-protons there is the problem of low intensity and secondary particles. Using reverse protons would identify any large discrepancies between BPM and magnet centers.

\subsection{Try to Fix Possible $\pi$-Bumps}

The experiment described in Section 5.2 is quick and can be done parasitically. Although we doubt that a large fraction of the aperture restriction is caused by $\pi$-bumps, if there is a restriction from them, this simple experiment will fix the problem.

\subsection{Try Dispersion Bumps}

We have studied the possibility of creating closed orbit bumps that produce dispersion and a slope of the dispersion at the kicker. Unfortunately the knobs that we found do not create a very large dispersion wave while creating a sizable orbit distortion and they are limited by the maximum strength of some of the corrector magnets. Nevertheless we recommend trying the knobs. The test can be done parasitically.

\subsection{Matching the On-Energy Lattice Functions at the Lithium Lens}

This is another parasitic experiment that might not improve the transmission at all, but if there is a mismatch, one could gain. It should not be very difficult to calculate knobs to change the $\alpha$ - and $\beta$-functions after the lithium lens, leaving the lattice functions at the end of the line as they are and not changing the ones in between to too large values. Once the knobs are calculated, one can use them as a tuning tool. 


\subsection{Rematching the Lattice}

If the recommendations described so far have not increased the aperture to a sufficient value, one has to rematch the lattice for better off-momentum behavior. This should be done in stages and after each stage the possible aperture gain should be evaluated. It might be sufficient to do only the first step, but it might also be necessary to do them all in order to get sufficient aperture.

At first, one should rematch the lattice changing only the strengths of the quadrupole magnets. This should decrease the phase advance and chromaticity as well as the chromatic $\beta$-functions. This will of course increase the on-energy $\beta$-functions but at most places there is sufficient aperture to tolerate a moderate increase.

If this does not increase the aperture sufficiently, one can allow the matching routine to move quadrupole magnets. Perhaps it might be helpful to add some quadrupole magnets, but that needs to be studied.

If one still needs more aperture, one needs to study adding sextupole magnets to the line. These could either be separate magnets or shims on dipole magnets, or both. One would need to evaluate where one needs to put them and what might be required to accommodate them (as they must be located in an area with dispersion, which in this line is very crowded with dipole magnets, one might have to use shorter but stronger dipole magnets in order to make room for the sextupole magnets or one would need to build a chicane in a less crowded area).

If even with sextupole magnets the aperture is not sufficient, octupole magnets might be needed.

\subsection{Additional Correctors and Monitors}

More correctors and monitors would definitely be helpful. However we think first one should evaluate rematching the lattice to reduce the total phase advance significantly (thereby requiring fewer monitors and correctors). This might also change the phase advance between elements to more beneficial values.

\subsection{Study Multipole Errors}

The multipole errors of the magnets in the line are not well known. We have not yet studied their influence on the beam dynamics. However, due to the large emittance, a significant fraction of the beam particles travel 
through the magnets at large amplitudes. We therefore think multipole errors should be included in future studies. This might require measuring the multipole fields on several of the magnets in the line (so far only one of each type of magnet has been measured [2]). This might be especially important for the three quadrupole magnets in the injection channel where the injected beam travels far off center.

\section{Issues Currently under Study}

We are currently working on the following issues:

- Rematching the lattice for a lower phase advance: The current phase advance is of the order of 4 and we hope to get it down by one unit. As this should overall weaken the strength of the quadrupole magnets this should improve the chromatic behavior of the line.

- Adding sextupole magnets to improve chromatic behavior: If lowering the phase advance does not lower the chromatic functions sufficiently (and the probability for that is very high), sextupole magnets need to be added to the line at a place with dispersion. As the dispersive parts of the line are too crowded to add magnets, we are looking at different options to create dispersion in less crowded areas of the line.

- Field errors in Q405: The beam is going off center by more than $10 \mathrm{~cm}$ in that magnet. We are currently working on simulating what the actual field is in that area. We will then incorporate the result in the lattice to study the influence on the beam. A magnet similar to Q405 has recently been measured [2]. The results have not yet been analyzed.

- Non-closure of one of the $\pi$-bumps: We are looking at the measurement results that showed a non-closure of one of the local orbit bumps. We are trying to understand if the non-closure was simply caused by the phase advance being not exactly $\pi$ even in the model or if it is an indication of a significant difference between model and machine in that area.

- Adding corrector magnets: Even after lowering the phase advance the correctors might still be at unfortunate phase advances and there might still not be enough of them in some areas of the line. As soon as 
we have a lattice for lower phase advance we will look at how many additional corrector magnets are required and where they should be located.

- Tracking through the beamline and the Debuncher: As soon as we are sure what the positions and strengths of the injection channel quadrupoles are we will do more tracking studies to see, if the aperture restriction is actually in the Debuncher and not in the line. This also requires putting the apertures from the Debuncher into the model used for tracking which is currently under way.

There are also a few issues worth studying that we might look at in the future:

- Local orbit bumps in small apertures.

- Multipole Errors in all magnets.

- Knobs to match the on-energy lattice functions at both ends of the line empirically.

\section{Summary}

Our studies have shown that the aperture problems of the AP2 beam line are not so much a problem of a single element not having enough physical aperture but more a problem of chromatic effects and imperfections. Some of the recommendations require simply a few hours (or less) of machine time and we suggest that these be done as soon as possible. They would either directly improve the aperture and therefore the transmission or they would show that what we assumed to be a possible problem is actually not a problem.

Once those experiments have been done and the different stages of rematching the lattice have been studied, one can evaluate what modifications to the beam line are desired.

\section{Acknowledgments}

We would like to thank Keith Gollwitzer, Elvin Harms and Steve Werkema for providing lots of information in order to do the studies presented here and for many useful discussions. We would also like to thank Keith and Steve for their hospitality during our visit and the tour of the AP2 beamline 
and the Debuncher which was really helpful. We would also like to thank John Corlett, John Jowett, Christoph Steier, André Verdier, Andy Wolski and Mark Woodley for helpful discussions.

\section{References}

[1] Design Report, Tevatron I Project. Technical report, Fermilab, September 1984.

[2] S. Werkema, private communication.

[3] Keith Gollwitzer and Steve Werkema. AP2 and Debuncher Acceptance Upgrades. Pbar Note 680, Fermilab, April 2003.

[4] M. Church. Recent AP2 studies and a proposal to increase the transverse aperture to 40 pi-mm-mrad. Pbar Note 571, Fermilab, October 1997.

[5] F. Bieniosek. Increasing the transverse aperture of the AP2-Debuncher injection region. Pbar Note 595, Fermilab, June 1998.

[6] H. Grote and F. C. Iselin. The MAD Program, User's Reference Manual. SL Note 90-13 (AP) (Rev. 5), CERN, April 1996.

[7] K. Gollwitzer, private communication.

[8] M. Woodley, private communication.

[9] A. Wolski, private communication. 